\title{
Análisis del modelo de juego en un equipo de fútbol profesional de la Bundesliga de Alemania. Estudio caso \\ Analysis of the game model in a professional football team in the German First Division. Case study

\author{
*Alberto Martín Barrero, **Isaac Marcos Gutierrez, **Moisés Falces Prieto
} \\ *Centro Universitario San Isidoro (España), Academia Alto Rendimiento Marcet (España)
}

\begin{abstract}
Resumen: La tendencia en los últimos años de querer perfeccionar las estrategias y planteamientos tácticos ha hecho que el fútbol evolucione hacia nuevos enfoques que permitan analizar y elaborar planes estratégicos mucho más profundos y rigurosos. Para ello se han establecido planes estratégicos como el modelo de juego, el cual pretende establecer los patrones de juego claves de un equipo con el objetivo de poder desarrollar una estructura que identifique y defina los rasgos de un equipo. Este trabajo tiene como objetivo realizar un estudio caso que analice el modelo de juego de un equipo de fútbol profesional de Alemania mediante la visualización de todos sus partidos de liga ( $\mathrm{n}=25$ ) antes del COVID-19 y utilizando una metodología descriptiva, estableciéndose 2 criterios y 4 elementos de análisis. Los resultados indican, en líneas generales, que el equipo analizado es un equipo marcado por un patrón combinativo de juego ofensivo, que trata de desequilibrar al rival mediante progresión por la zona central del campo y que a nivel defensivo se define por ser un equipo que intenta recuperar el balón lo antes posible, tapando los pasillos interiores, pudiéndose concluir cómo a través de un estudio caso podemos analizar y estudiar las características de un equipo de fútbol.

Palabras clave: fútbol, análisis táctico, modelo de juego, estudio caso, rendimiento.
\end{abstract}

\begin{abstract}
The trend in recent years of wanting to perfect strategies and tactical approaches has led football to evolve into new approaches that allow for much deeper and more rigorous strategic plans to be analyzed and developed. For this, methods such as the game model have been established, which aims to establish the key game patterns of a team in order to develop a structure that identifies and defines the characteristics of a team. The objective of this work is to carry out a case study that analyzes the game model of a professional soccer team in Germany by visualizing all its league games $(\mathrm{n}=25)$ before COVID-19 and using a descriptive methodology, establishing 2 criteria and 4 elements of analysis. The results indicate, in general terms, that the team analyzed is a team marked by a combinative pattern of offensive play, which tries to unbalance the rival by progression through the central area of the field and which, at a defensive level, is defined as a team that tries to recover the ball as soon as possible, covering the interior corridors, being able to conclude how through a case study we can analyze and study the characteristics of a soccer team.
\end{abstract}

Key words: soccer, tactical analysis, game model, case study, performance.

\section{Introducción}

En los últimos años se han publicado un gran número de artículos relacionados con el fútbol (Cabrera-Domene \& Falces-Prieto, 2019), convirtiéndose en una materia de estudio desde un punto de vista multidisciplinar (Martín-Barrero \& Martínez-Cabrera, 2019). Podemos encontrar numerosos trabajos e investigaciones relacionadas con la planificación metodológica (Arjol, 2012), acciones a balón parado (Pérez \& Fonseca, 2015), evolución ofensiva (Barreira, Garganta, Castellano, Prudente, \& Anguera-Argilaga, 2014), modelos de juego (Martín-Barrero, 2016), lesiones deportivas (Noya \& Sillero, 2012), juegos reducidos (Falces-Prieto et al., 2015), análisis del rendimiento desde el plano de la condición física (Pérez-Martínez \& Torrebadella-Flix, 2017) y desde la perspectiva psicológica (Navarrón, Godoy-Izquierdo, Vélez, Ramírez-Molina, \& Jiménez-Torres, 2017) entre otros. Sin embargo, en los últimos tiempos, son numerosos los autores e investigadores que han pasado a analizar el rendimiento en fútbol desde una perspectiva táctica (Drust, Atkinson, \& Reilly, 2007; Perl, Grunz, \& Memmert, 2013; Reilly, 2005). La fluctuación de los comportamientos que se dan durante el juego es una realidad difícil de explicar y predecir (Castellano, 2008) y es aquí donde el análisis técnico-táctico tanto de jugadores como de equipos entran en valor (Fenoll-Espejo,

Fecha recepción: 07-05-20. Fecha de aceptación: 04-09-20

Alberto Martín Barrero

amarbar10@gmail.com
Martínez-Sánchez, \& Cara-Muñoz, 2019). Por lo tanto, el fútbol ha pasado de analizarse desde una perspectiva y metodología reduccionista a una metodología que comprende la globalidad de los factores y momentos que componen el fútbol (Tamarit, 2010), y, por tanto, entendida desde un prisma que intente plasmar en el entrenamiento las situaciones reales de juego (Martín-Barrero \& Martínez-Cabrera, 2019).

Dentro de las variables de estudio técnico-tácticas colectivas, encontramos el análisis del modelo de juego (MJ) (Bettega et al., 2016). Son numerosos los técnicos, entrenadores e investigadores que tratan de conceptualizar y expresar el significado sobre el MJ (Cano, 2012; Delgado \& Méndez-Villanueva, 2012; Guindos, 2015; Martín-Barrero, 2016; Oliveira, 2004; Tamarit, 2010). Nos obstante, en el contexto del fútbol aún hay cierta confusión en relación a este concepto. Por ello, debemos contextualizar o definir qué es el MJ. En relación al MJ, Oliveira (2004) lo entiende como una idea/conjetura de juego constituida por principios, sub-principios, sub-principios de los sub-principios, etc., representativos de los diferentes momentos / fases de juego, que se articulan entre sí, manifestando una organización funcional propia, es decir, una identidad. Por su parte, Mourinho (2014) reafirma esta característica de identificación, definiéndolo como los principios que establezco en mi equipo, los principios prioritarios que nos otorga un cierto $\mathrm{ADN}$ (una identidad). La organización de los elementos y componentes que conforman el juego, junto a la longevidad o regularidad de la misma, es una propiedad a resaltar en la definición de MJ (Martín-Barrero \& Martínez-Cabrera, 2019). Para Amieiro (2005), el MJ o la identidad de un equipo no es más que la 
afirmación regular de la organización que conforman. En este sentido, Tamarit (2010) indica que se trata de la organización que presenta un equipo en cada momento del juego y lo hace de forma regular. En relación a la prolongación en el tiempo, Frade (1985) indicó que el MJ debe de estar siempre en entre dicho, manteniéndose el futuro como elemento causal del comportamiento.

Otra característica importante sobre el MJ que encontramos analizando la bibliografía, es la importancia del jugador y sus interacciones dentro de este modelo u organización (Martín-Barrero \& Martínez-Cabrera, 2019). Para Cano (2012), el MJ es la organización de las tendencias conceptuales que se originan con la interacción de las capacidades naturales de quienes juegan. Aunque bien es cierto que el jugador es o debe ser el principal protagonista de un MJ, este debe ser diseñado, guiado y construido por parte del entrenador, el cual juega un rol crucial en este escenario (Martín-Barrero \& Martínez-Cabrera, 2019). Como uno de los últimos elementos que caracteriza al MJ encontramos la flexibilidad del mismo, ya que este debe permitir adaptarse a diferentes contextos y ser abierto según los jugadores y circunstancias de juego (Martín-Barrero, 2018). Por su parte, Freitas (2004) cita que el entrenador debe llevar y presentar a los jugadores una imagen del MJ en sus cabezas, constatando que el entrenador debe tener un rol muy importante en el diseño del entrenamiento y en la configuración de las tareas del mismo. Además de esta función, el entrenador debe permitir que el MJ desarrolle la capacidad de aprender a aprender, es decir, que lleve al jugador a autogestionar su proceso de entrenamiento y aprendizaje dentro del mismo (Martín-Barrero, 2018), ya que, el juego en el fútbol se caracteriza por su dinamismo y complejidad, estableciendo un ambiente caótico, en el cual emergen posibilidades de acción en todo momento (Bettega et al., 2016).

Toda la información relevante al estudio de los MJ y el óptimo desarrollo del mismo, viene precedido por el análisis y la evaluación. Así pues, la evaluación táctica ha sido realizada por medio de tecnologías avanzadas, tales como AMISCO o y Prozone ${ }^{\circledR}$ (Castellano, Álvarez-Pastor, \& Bradley, 2014), uso de sistemas globales de posicionamiento (GPS) (Tierney, Young, Clarke, \& Duncan, 2016), información mediante datos posicionales (Memmert, Raabe, Schwab, \& Rein, 2019) y el uso del Big Data (Rein \& Memmert, 2016), entre otras. Todas estas herramientas son usadas bajo los parámetros y criterios del análisis de juego (González-Víllora \& Costa, 2015). Estos sistemas de análisis son demasiado complejos y presentan costes elevados, sólo aptos para el fútbol profesional. Sin embargo, una manera práctica, con pocos recursos humanos, económica y no invasiva de evaluar en fútbol, es el uso de la evaluación observacional (González-Víllora \& Costa, 2015). Este tipo de evaluación ha sido ampliamente utilizada en fútbol (Castellano, Mendo, De Segura, Fontetxa, \& Bueno, 2000; Paulis \& Mendo, 2000). La metodología observacional (MO) es una estrategia del método científico que plantea la observación sistemática de la realidad sin alterarla y que tiene como objetivo general el análisis de la conducta espontánea de un participante o grupo en un determinado entorno (Anguera \& Mendo, 2013), desarrollando unas categorías que permitirán obtener registros sistemáticos de la conducta, que una vez transformados en datos cuantitativos con un nivel de fiabilidad determinado, permitirán describir y en algunos casos predecir el comportamiento de los sujetos en las situaciones analizadas (Sanmiguel-Rodríguez, 2020). Esto convierte a la MO en la más idónea para el estudio del deporte, cuando el objetivo es analizarlo en su contexto y dinámica habitual (Anguera \& Mendo, 2015).

Con toda esta información, indicamos que el objetivo de este estudio fue analizar el modelo de juego de un equipo de fútbol profesional de la Bundesliga alemana utilizando la metodología observacional para analizar los partidos disputados antes del COVID-19 durante la temporada 2019/2020. Esta temática es transcendental ya que podría contribuir al desarrollo del fútbol con respecto a estudios focalizados en la perspectiva táctica del juego.

\section{Método}

Se utilizó el estudio caso descriptivo (Baxter \& Jack, 2008; Chaverra, Gaviria \& González, 2019) como metodología cualitativa y de indagación naturalista (Pérez, 2008), la cual trata de investigar empíricamente un fenómeno contemporáneo dentro de un contexto de la vida cotidiana (Yin, 1984). Para establecer criterios de rigor y credibilidad se siguió la propuesta de Guba (1989) para validar sus hallazgos y conclusiones, basadas en a) realizar una descripción detallada sobre cómo fue recogida y analizada la información y b) en qué contexto se realiza la investigación y cómo se llevó a cabo el análisis (Tabla I).

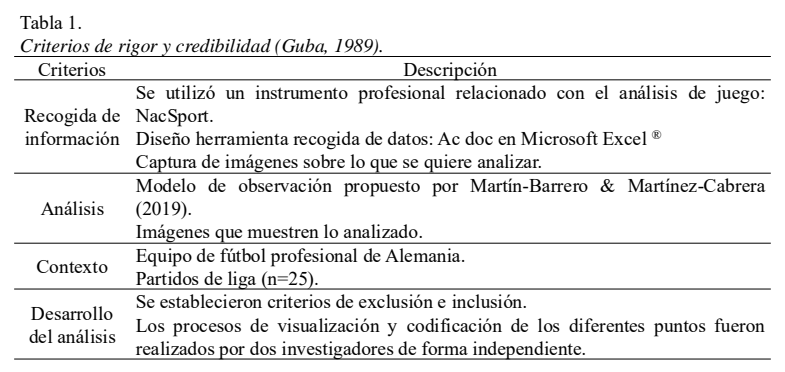

Se analizó el modelo de juego de un equipo de fútbol profesional de la primera división de Alemania (RB Leipzig), durante todos los partidos $(n=25)$ de la temporada 2019-2020 antes del inicio del COVID-19. Para el análisis del modelo de juego se utilizó como instrumento de recogida de datos el software NacSport en su versión Pro+ para Windows, a la misma vez que se complementó con una herramienta Ac doc a través de Microsoft Excel ${ }^{\circledR}$ diseñada para ir desarrollando los diferentes apartados (Figura 1). Para finalizar el apartado de recogida de datos se decidió capturar diferentes imágenes que ayuden a dar claridad a lo que se observa. Como instrumento de observación y análisis se utilizó la propuesta Martín-Barrero \& Martínez-Cabrera (2019) para diseños de modelos de juego, en la cual establecen 2 criterios claves (formales y funcionales) y 4 elementos (criterios de inclusión) para elaborar, analizar y desarrollar un modelo de juego en el fútbol.

\section{Criterios de inclusión}

Estilo de juego: comportamientos generales llevados a cabo por el equipo y que le otorgan una identidad. 
Patrones de juego: principios y subprincipios del juego llevado a cabo en cada fase y momento del juego.

Sistemas de juego: posicionamiento y organización espacial de los jugadores en el terreno de juego.

Ciclo del juego: para definir los comportamientos en cada una de las fases seguiremos la propuesta de organización del juego divida en dos fases: ataque y defensa, y cuatro momentos del juego: ataque organizado, contraataque, organización defensiva y defensa organizada (Cano, 2012; Martín-Barrero \& Martínez-Cabrera, 2019).

\section{Criterios de exclusión}

Con el fin de concretar y sintetizar los aspectos que más caracterizan al modelo de juego del equipo analizado, de la propuesta de Martín-Barrero \& Martínez-Cabrera (2019) se decidió excluir por parte de los investigadores los siguientes puntos de los elementos formales:

- Plantilla.

- Objetivos secuenciales del juego.

$\mathrm{Y}$ los siguientes puntos de los elementos funcionales: ABP.

Funciones específicas por puestos.

Patrones de juego a adoptar ante estrategia rival.

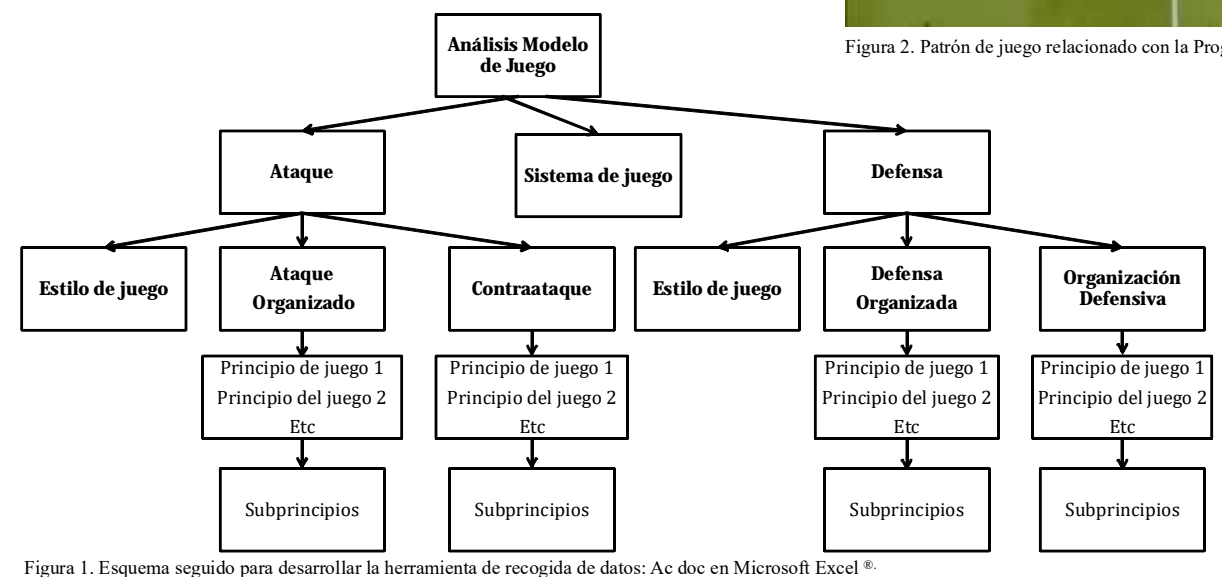

o Patrones de juego: se establecerán patrones de juego generales (Tabla II y Tabla III) y patrones de juego específico, ambos por fase y momento del juego.

\section{Ataque Organizado}

- Progresión en el juego:

o Tienen la intención de generar ventajas desde los primeros pases, es el único momento en que el equipo alemán se toma un momento de pausa, busca que sus centrales lleguen lo más arriba posible con balón antes de buscar un pase vertical hacia líneas más adelantadas, no tienden a dar muchos pases horizontales en este momento del juego, identifican muy bien las acciones de pases para atraer y pases para eliminar líneas. Cuando centrales buscan ser verticales

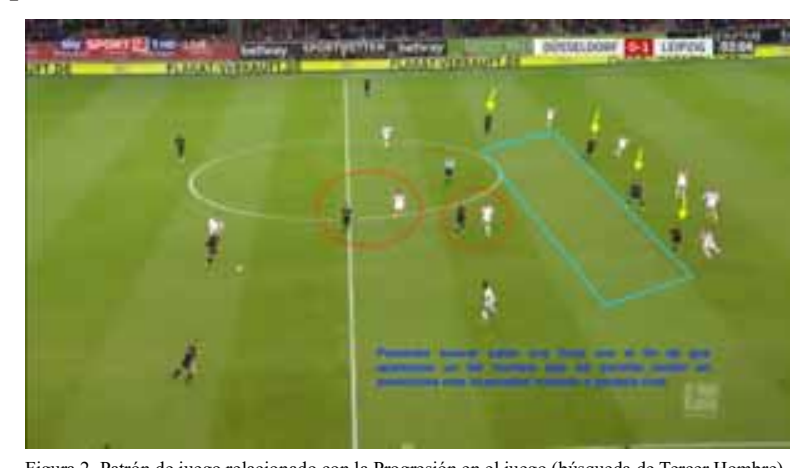

con sus pases, normalmente esos pases son saltando la línea inmediata (pivotes), buscando última línea (puntas) o una línea anterior (interiores/media puntas) con el fin de poder generar terceros hombres en campo rival, situarse de cara a la portería rival y poder aumentar el ritmo de juego ofensivo.

\section{Resultados}

\section{Elementos formales}

o Sistemas de juego: el equipo analizado se caracteriza por utilizar hasta 5 sistema de juego diferentes:
1-4-2-2-2 (usado en el 52\% de los partidos)
1-3-1-4-2 (usado en el $24 \%$ de los partidos)
1-3-4-3 (usado en el 12\% de los partidos).
1-3-4-2-1 (usado en el 8\% de los partidos).
1-4-2-3-1 (usado en el $4 \%$ de los partidos).

\section{Elementos funcionales}

o Estilo de juego: el estilo de juego ofensivo del equipo se caracteriza por ser combinativo o indirecto (Ruiz-Ruiz, Fradua, Fernández-García \& Zubillaga, 2013) y a nivel defensivo se caracteriza principalmente por presión alta (Wright, Atkins, Polman, Jones \& Sargeson, 2011).

Tabla 2

Tabla 2.
Patrones de juego de la fase ofensiva.
\begin{tabular}{ll}
\multicolumn{1}{c}{ Ataque Organizado } & \multicolumn{1}{c}{ Contraataque } \\
\hline a) Progresión en el juego. & a) Ocupación de diferentes pasillos. \\
b) Relación de ocupación de espacios. & b) Progresar o lanzar. \\
c) Acumulación de jugadores en área rival. & c) Llegadas zonas de remate. \\
\hline
\end{tabular}
- Relación de ocupación de espacios:

o Para construir situaciones de ataque y finalización, aparecen diferentes elementos que facilitan la verticalidad y un ritmo alto asociándose con balón en campo rival, elementos como: ocupación de todos los pasillos (interiores, exteriores y central, dividiendo el campo en 5 pasillos verticales), posicionamiento a diferentes alturas, marcando diferentes roles ofensivos en los jugadores, fijadores interlinea; jugadores que se posicionan en espacios intermedios dentro de la misma línea, fijadores por fuera: jugadores que se mantienen dando amplitud al equipo y por ultimo jugadores relacionándose cerca de balón: se acercan a poseedor con el fin de poder ser el segundo hombre en las continuas acciones de tercer hombre que realiza el conjunto alemán o para permitir dar continuidad a la posesión de balón. Todo con el fin de poder generar espacios entrelineas y provocar pasillos de penetración. Es raro ver los jugadores que parten en una posición en el momento de la salida de balón mantengan esa posición cuando el balón llega a zonas más avanzadas, esos jugadores suelen identificar, su relación respecto al juego: si son cercanos o alejados en la jugada y se mueven en conso- 
nancia, siendo opción de pase para dar continuidad a la jugada o buscando pase en la línea más adelantada, si son cercanos o manteniéndose alejados buscan continuamente generar una línea de pase que les permita mirar a portería rival a todo su equipo a través de un tercer hombre o conduciendo intentando llegar a última línea rival si recibe con espacio.

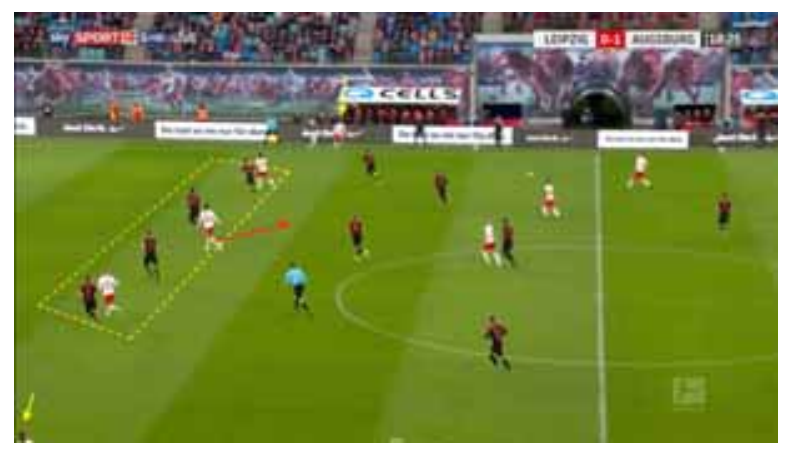

Figura 3. Patrón de juego relacionado con la Ocupación de espacios (fijadores interlínea).

- Acumulación de jugadores en área rival:

o El hecho de que el equipo alemán sea un equipo muy vertical y muy dinámico en campo rival, provoca que lleguen al área rival con varias opciones de remate ante pase filtrado o centro lateral, ocupan todas las zonas de remate, potenciando zonas de punto de penalti más la importancia de poder ganar una $2^{\circ}$ jugada ante un centro rival. No es raro ver al equipo alemán llegar hasta con 6 jugadores en zonas de remate.

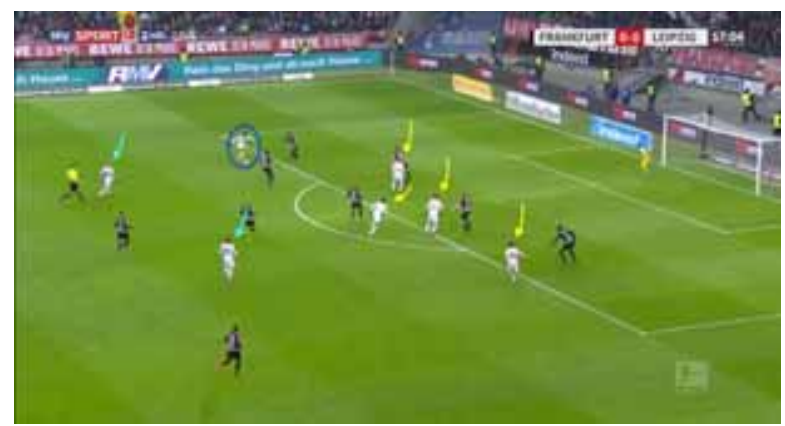

Figura 4. Patrón de juego relacionado la acumulación de jugadores en área rival (ocupación de zonas de remate)

\section{Contraataque}

- Progresar o lanzar:

Identifican muy bien el momento de la recuperación de balón, es uno de los puntos que el RB Leipzig tiene muy en cuenta a la hora de realizar la transición defensa - ataque, ¿el cómo recuperó el balón? de cara a portería rival, bien perfilados o de espaldas a portería rival, mal perfilados. En función

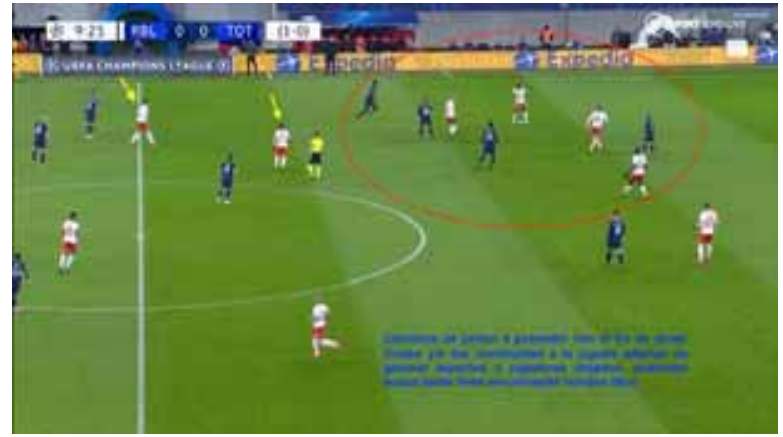

Figura 5. Patrón de juego relacionado con progresar o lanzar (búsqueda de hombre libre tras atraer). de esa acción el conjunto alemán actúa de una manera u otra, donde independientemente de cómo se encuentren en el momento del robo, el objetivo es encontrar un pase vertical a un jugador adelantado que deje de cara para permitir que el receptor de ese pase este de cara a portería rival y con espacio para decidir si ser conductor de contra o pasar a jugadores que se encuentran desmarcándose a los espacios.

- Ocupación de diferentes pasillos.

o Una vez consiguen salir de la presión tras perdida que intentan ejercerle los rivales, el RB Leipzig transita a una manera muy rápida, con una percepción de pasillos a ocupar muy alta, indistintamente de la posición de los jugadores, cuando roban y tienen espacio la única misión de los jugadores que participan en ese momento de contraataque más allá de llegar al área rival y finalizar con éxito, es la de ocupar un pasillo que no este ya ocupado por un compañero, con el fin de poder dar más opciones al poseedor de balón que se encuentra lanzando la transición.

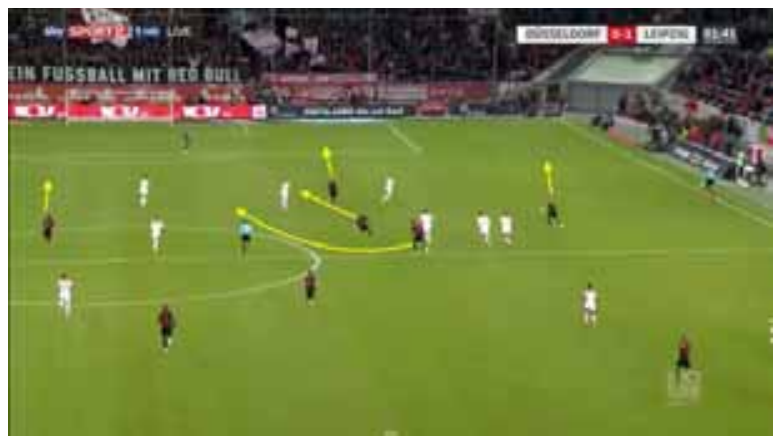

Figura 6. Patrón de juego relacionado con ocupación de diferentes pasillos(ocupar pasillos desocupados)

- Llegadas a zonas de remate.

o El hecho de que sea una transición defensa - ataque, un momento que dura escasos segundos donde se aprovecha la desorganización rival y donde actúan los jugadores mejor preparados después del momento de la recuperación de balón, no es indiferente para que el RB Leipzig llegue con muchos jugadores al área rival y gestionen de manera organizada como ocupar las zonas de remate.

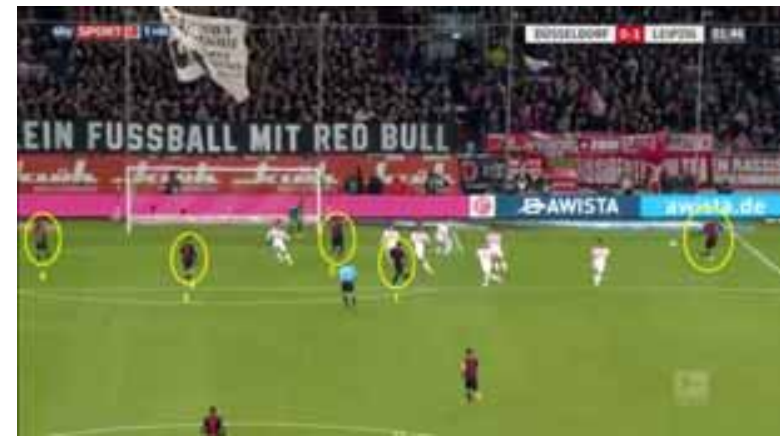

Figura 7. Patrón de juego relacionado con llegada a zonas de remate (ocupación racional del área).

Tabla 3.

Patrones de juego de la fase defensiva.

\begin{tabular}{ll}
\hline \multicolumn{1}{c}{ Defensa Organizada } & \multicolumn{1}{c}{ Organización defensiva } \\
\hline a) Equipo en bloque Alto en la salida rival. & a) Presión tras perdida. \\
b) Orientar la progresión del rival. & b) Temporización y repliegue.
\end{tabular}

b) Orientar la progresión del rival.

c) Reducir espacio carriles interiores y carril central. c) Vigilancias defensivas.

\section{Defensa Organizada}

- Equipo en bloque Alto en la salida rival:

oEL RB Leipzig no es un equipo que destaque especial- 
mente por su intensidad en el momento de presión alta en la salida de balón rival, dejan que centrales rivales tengan de forma más o menos calmada el balón en su inicio, su objetivo es tapar las opciones de pase en corto para obligar al rival a jugar directo, sabedores de que su línea defensiva destaca en los duelos individuales, si no consiguen que su rival juegue de manera directa, entonces buscan que la salida de balón rival sea orientada hacia un carril exterior. Esta es la primera intencionalidad que tienen los jugadores más adelantados del conjunto alemán.

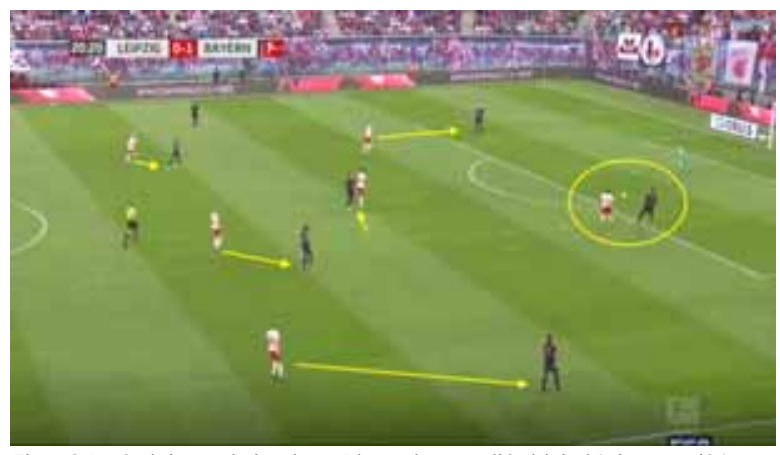

Figura 8. Patrón de juego relacionado con Bloque alto ante salida del rival (orientar presión).

- Orientar la progresión del rival:

oPriorizan tapar pasillos interiores y pasillo central en fase defensiva, estando muy juntos entre líneas, jugadores próximos a balón suelen evitar la entrada para no ser superados y desajustar las líneas de su equipo, más bien son un equipo que busca llevar al rival a las zonas más provechosas para poder robar el balón, indistintamente si es en una zona u otra del campo, sobre todo buscan orientar al rival hacia carriles exteriores donde hay menos espacio para, a partir de un buen sistema de ayudas defensivas, robar y transitar.

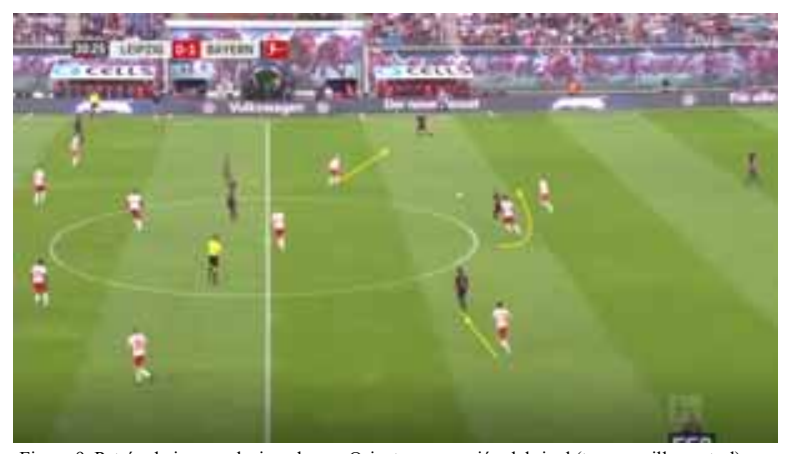

Figura 9. Patrón de juego relacionado con Orientar progresión del rival (tapar pasillo central).

oEn los momentos sin balón, igual que identifican muy bien las situaciones de llevar el bloque arriba para presionar

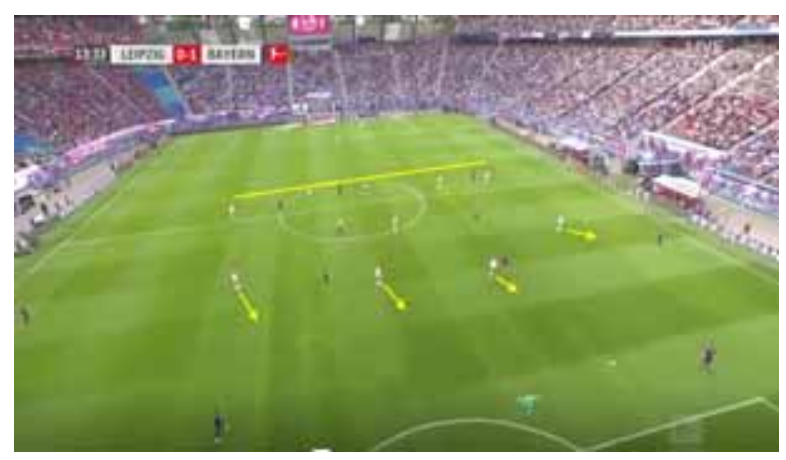

Figura 10. Patrón de juego relacionado con Orientar progresión del rival (ubicación en bloque medio). la salida de rival, también saben identificar los momentos que el juego ofensivo del rival les lleva a realizar un bloque medio, también es el momento defensivo en el que se sienten más cómodos, priorizando dejar muy poco espacio entre líneas y evitando constantemente la posible aparición de intervalos entre jugadores de la misma línea, con estos comportamientos con el bloque en medio campo intentan llevar al rival hacia zonas exteriores, lugar donde como hemos comentado llevan al rival para provocar la recuperación de balón.

\section{Organización defensiva}

- Presión tras pérdida

o No destacan por tener un excesivo cambio de ritmo en el momento de la perdida, activándose sobre poseedor y posibles opciones con una intensidad en el esfuerzo elevada para provocar la recuperación de balón lo antes posible y con ello lo más cerca de portería rival, no podrían basar tu transición ataque - defensa a una acción así por su forma de atacar, básicamente realizan una presión tras perdida muy inteligente donde acumulan jugadores en la zona activa donde se produjo la perdida y el jugador más cercano a poseedor lo encima pero sin realizar ningún tipo de entrada, evitando totalmente ser superado ya que su objetivo primordial es evitar que el rival pueda activar un pase que le permita lanzar el contraataque.

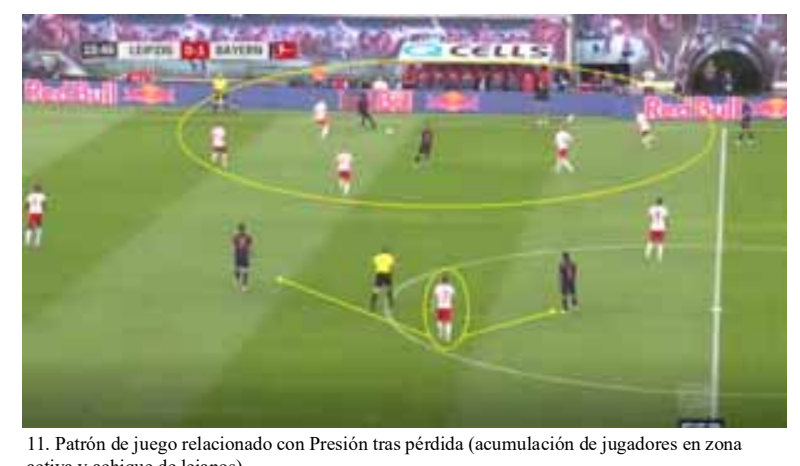

activa y achique de lejanos).

- Temporización y Repliegue.

o Son un equipo donde suelen mostrar una tendencia de este tipo de acciones llevándolas a cabo en campo propio, ya que por su forma de atacar no logran siempre poder robar con éxito en campo rival, por lo que la mayoría de estas acciones las realizan jugadores de líneas atrasadas con perfiles más defensivos, que dominan este tipo de acciones a la perfección, también muchas de estas acciones las generan en campo abierto y con muchos jugadores propios eliminados respecto a la línea de balón.

- Vigilancias defensivas.

o Como llevamos viendo en toda la faceta defensiva, ya sea en el momento de defensa organizada o el momento de transición ataque - defensa, son un equipo que su forma de defender viene precedida por la forma en la que atacan, esto hace que sus centrales, ya jueguen con línea de 4 o con línea de 3, dominen perfectamente este tipo de acción, posicionándose de manera adecuada para sacar ventajas en los posteriores duelos defensivos, ya sean aéreos o en carrera a campo abierto. 


\section{Discusión y conclusiones}

El fútbol profesional requiere cada vez más de instrumentos que ayuden a analizar a los diferentes equipos desde una perspectiva multidisciplinar (González, Campos \& Romero, 2014; Guedea-Delgado, Nájera-Longoria, NúñezEnríquez, Candía-Luján, \& Gastélum-Cuadras, 2019) con el fin de optimizar su rendimiento en competición. Saber analizar el modelo de juego de un equipo permite sostener ese análisis sobre una estructura que aporte rigor y valor a lo observado (Guindos, 2015).

A través del desarrollo y análisis del modelo de juego del RB Leipzig hemos observado que es un equipo que tiene un modelo de juego caracterizado unos rasgos generales (Tabla IV) y por un estilo combinativo en ataque, el cual está basado en encontrar hombres libres y tercer hombre en los pasillos interiores del terreno de juego. Por otra parte, en relación al estilo defensivo, se caracteriza por ser un equipo que suele ser atrevido e intenta robar el balón en el campo contrario, adelantando las líneas y provocando el error del rival.

\begin{tabular}{ll}
$\begin{array}{l}\text { Tabla } 4 . \\
\text { Rasgos generales del Modelo de juego. }\end{array}$ & \multicolumn{1}{c}{ Rasgos generales defensivos } \\
\hline \multicolumn{1}{c}{ Rasgos generales ofensivos } & \multicolumn{1}{c}{} \\
\hline $\begin{array}{l}\text { Relación de jugadores a través de la } \\
\text { posesión del balón. }\end{array}$ & $\begin{array}{l}\text { Equipo en bloque alto con el fin de robar el balón } \\
\text { en campo contrario. }\end{array}$ \\
\hline $\begin{array}{l}\text { Progresión hacia zonas avanzadas buscando } \\
\text { hombres libres en pasillo interior. }\end{array}$ & $\begin{array}{l}\text { Orientación del juego rival hacia los pasillos } \\
\text { exteriores. }\end{array}$ \\
\hline $\begin{array}{l}\text { Llegada al área con acumulación de } \\
\text { jugadores. }\end{array}$ & $\begin{array}{l}\text { Presión tras pérdida de la posesión con el fin de } \\
\text { recuperar la misma lo antes posible. }\end{array}$ \\
\hline
\end{tabular}

Por otra parte, el uso de diferentes sistemas de juego puede ayudar a entender ciertos criterios tácticos (Pascual, 2012; González, 2013). Analizando la gestión que realiza sobre el uso de sistemas de juegos, sería interesante resaltar que es un equipo que es capaz de adaptarse a las características de su plantilla y optimizar sus recursos en relación a las necesidades del equipo y las posibilidades de rendimiento del mismo con las oportunidades y debilidades que pueda ofrecer el rival. Este aspecto es muy importante, sobre todo en aquellos equipos que no cuentan con un presupuesto del más alto nivel. Por lo tanto, el modelo de juego del RB Leipzig tiene unos patrones bien definidos y consolidados tanto en la fase ofensiva del juego como en su fase defensiva, identificándose claramente en cada uno de los momentos del juego las intencionalidades tácticas que pretende desarrollar. Esta conclusión nos hace indicar que este equipo cuenta con una estructura de juego consolidada y sustentada en las virtudes del propio equipo, así como su capacidad de adaptación a las posibles situaciones que el rival pueda plantearle desde el plano estratégico.

Por último se puede concluir, tras el desarrollo de este trabajo, que sería interesante considerar al estudio caso como una alternativa metodológica (Chaverra, Gaviria \& González, 2019) que ayude a los entrenadores y técnicos en el análisis del modelo de juego en equipos de fútbol, siempre y cuando esté dentro de unos criterios que aporten veracidad y fiabilidad a las diferentes observaciones que se realicen. Como futuras líneas de investigación se propone el analizar todos los elementos que configuran un Modelo de Juego planteados en Martín-Barrero \& Martínez-Cabrera (2019) en el desarrollo de la temporada de un equipo de fútbol profesional.

\section{Referencias}

Amiero, N. (2005). Defensa en zona en el fútbol. Editorial McSport, Barcelona, España.

Anguera, M. T., \& Mendo, A. H. (2013). La metodología observacional en el ámbito del deporte. Ebalonmano.com: Revista de Ciencias del Deporte, 9(3), 135-160.

Anguera, M. T., \& Mendo, A. H. (2015). Técnicas de análisis en estudios observacionales en ciencias del deporte. Cuadernos de Psicología del Deporte, 15(1), 13-30.

Arjol, J. L. (2012). La planificación actual del entrenamiento en fútbol.: Análisis comparado del enfoque estructurado y la periodización táctica. Acción Motriz, (8), 27-37.

Baxter, P., \& Jack, S. (2008). Qualitative Case Study Methodology: Study Design and Implementation for Novice Researchers. The Qualitative Report, 13(4), 544 559.

Bettega, O. B., Galatti, L. R., Schmitz Filho,A. G., Tozetto,A. V. B., Pérez, B. L., \& Scaglia, A. J. (2016). Planificación táctica en el fútbol: Aspectos generales y específicos. Ebalonmano.com: Revista de Ciencias del Deporte, 12(1), 45-52.

Cabrera-Domene, L., \& Falces-Prieto, M. (2019). Análisis conductual del saque de esquina en fútbol profesional. EmásF, Revista Digital de Educación Física, 10(58), 3546.

Cano, O. (2012). El juego de posición del FC Barcelona. Editorial McSport, Barcelona, España.

Castellano, J., Mendo, A. H., De Segura, P. G., Fontetxa, E., \& Bueno, I. (2000). Sistema de codificación y análisis de la calidad del dato en el fútbol de rendimiento. Psicothema, 12(4), 635-641.

Castellano, J. (2008). Análisis de las posesiones de balón en fútbol: frecuencia, duración y transición. Motricidad. European Journal of Human Movement, 21, 189-207.

Castellano, J., Álvarez-Pastor, D., \& Bradley, P. S. (2014). Evaluation of research using computerised tracking systems (Amisco ${ }^{\circledR}$ and Prozone ${ }^{\circledR}$ ) to analyse physical performance in elite soccer: A systematic review. Sports Medicine, 44(5), 701-712.

Chaverra, B., Gaviria, D. \& González, E. (2019). El estudio de caso como alternativa metodológica en la investigación en educación física, deporte y actividad física. Conceptualización y aplicación. Retos, 35, 422-427.

Delgado, J., \& Méndez-Villanueva, A. (2012). Tactical Periodization: Mourinho's best-kept secret? Soccer Journal, 57(3), 29-34.

Drust, B., Atkinson, G., \& Reilly, T. (2007). Future perspectives in the evaluation of the physiological demands of soccer. Sports Medicine, 37(9), 783-805.

Falces-Prieto, M., Casamichana, D., Sáez de Villarreal, E., Requena-Sánchez, B., Carling, C., \& Suárez-Arronez, L. J. (2015). The presence of the head coach during a smallsided game: effects on players' internal load and technical performance. RICYDE. Revista Internacional de Ciencias del Deporte, 11(41), 245-257.

Fenoll-Espejo, A.J., Martínez-Sánchez, J.A., \& Cara-Muñoz, J.F. (2019). Informe estadístico y técnico-táctico de un 
equipo de fútbol profesional de la primera división alemana. EmásF, Revista Digital de Educación Física, 60, 98-114.

Frade, V. (1985). ¿Alta competiçao no futebol-que exigencias do tipo metodológico? Facultad de Deporte de la Universidad de Porto, Porto, Portugal.

Freitas S. (2004). A especificidade que está na concentração táctica que está na Especificidade no que deve ser uma operacionalização da Periodização Táctica [monografía]. Universidad de Porto, Porto, Portugal.

Garganta, J. (2001). A análise da performance nos jogos desportivos. Revisão Acerca da Análise do Jogo, 1(1), 57-64.

Gónzalez, J. (2013). Análisis del rendimiento táctico en el juego colectivo ofensivo en fútbol en la Copa del Mundo 2010. Tesis doctoral. Valencia: Universitat de Valencia.

González, G., Campos, M. \& Romero, S. (2014). Análisis de la influencia de la evaluación del rendimiento en jugadores de un equipo de fútbol. Retos, 25, 85-89.

González-Víllora, S., \& Costa, I. T. (2015). ¿Cómo evaluar la táctica en fútbol? Sistema de evaluación de la táctica en fútbol (Fut-Sat). Educación Física y Deporte, 34 (2), $467-$ 505.

Guba, E. (1989). Criterios de credibilidad en la investigación naturalista. En J. Gimeno \& A. Pérez (Eds.), La enseñanza, su teoría y su práctica (pp. 148-165). Madrid: Akal.

Guedea-Delgado, J.C., Nájera-Longoria, R.J., Núñez-Enríquez, O., Candía-Luján, R., \& Gastélum-Cuadras, G. (2019). Sistemas tácticos y resultados de competición del Mundial de Fútbol Asociación de Rusia 2018. Retos, 36, 503-509.

Guindos, D. (2015). Construcción metodológica del modelo de juego. Editorial Futboldlibro, Madrid, España.

Hernández-Mendo, A., López-López, J.A., Castellano-Paulis, J., Morales-Sánchez, V., \& Pastrana-Brincones, J. L. (2012). Hoisan 1.2: Programa informático para uso en metodología observacional. Cuadernos de Psicología del Deporte, 12(1), 55-78.

Martín-Barrero, A. (2016). Modelo de juego: Leicester City FC: De la concepción teórica a la aplicación práctica. Revista Abfutbol, 82, 13-57.

Martín-Barrero, A. (2018). ¿Cómo construir el camino desde el fútbol de la calle al fútbol profesional? Editorial Abfutbol, Madrid, España.

Martín-Barrero,A., \& Martínez-Cabrera, F. I. (2019). El modelo de juego en el fútbol. De la concepción teórica al diseño práctico (Game models in soccer. From theoretical conception to practical design). Retos, 36(36), 543-551.

Martín-Barrero, A. \& Martínez-Cabrera, F. (2019). Fútbol: El modelo de juego. De la pizarra al entrenamiento. Editorial Wanceulen, España.

Memmert, D., Raabe, D., Schwab, S., \& Rein, R. (2019). A tactical comparison of the 4-2-3-1 and 3-5-2 formation in soccer: A theory-oriented, experimental approach based on positional data in an 11 vs. 11 game set-up. PloS one, 14(1).

Mourinho, J. (2014). The Daily Telegraph [entrevista]. Recuperado de https://www.telegraph.co.uk/

Navarrón, E., Godoy-Izquierdo, D., Vélez, M., Ramírez-Molina, M. J., \& Jiménez-Torres, M. G. (2017). Implementación de una intervención psicológica en fútbol base, satisfac- ción subjetiva de los deportistas y experiencias de pasión, competencia percibida y compromiso deportivo en relación con la intención de práctica futura. Revista Iberoamericana de Psicología del Ejercicio y el Deporte, 12(1), 59-69.

Noya, J., \& Sillero, M. (2012). Incidencia lesional en el fútbol profesional español a lo largo de una temporada: días de baja por lesión. Apunts. Medicina de l'Esport, 47(176), 115-123.

Oliveira, J.G. (2004). Conhecimento Específico em Futebol. Contributos para a definição de uma matriz dinâmica do proceso ensinoaprendizagem/ treino do jogo. Facultad de Deporte de la Universidad de Porto, Porto, Portugal.

Pascual, N. (2012). Análisis comparativo de los sistemas de juego en el fútbol 7. Tesis doctoral. Alicante: Universidad de Alicante.

Pérez, G. (2008). Investigación cualitativa. Retos e interrogantes I. Métodos ( $5^{\text {a }}$ Edición). Editorial La Muralla, Madrid, España.

Pérez, S., \& Fonseca, D. (2015). Influencia de las acciones a balón parado en el futbol de élite nacional e internacional: Análisis de los factores de competición y jugar como local o visitante. EmásF, Revista Digital de Educación Física, 32, 41-52.

Pérez-Martínez, C., \& Torrebadella-Flix, X. (2017). La preparación física del fútbol en España (1899-1930). Ebalonmano.com: Revista de Ciencias del Deporte, 13(2), 113-130.

Perl, J., Grunz, A., y Memmert, D. (2013). Tactics Analysis in Soccer-An Advanced Approach. International Journal of Computer Sciencie in Sport, 12(1), 33-44.

Reilly, T. (2005). An ergonomic model of the soccer training process. Journal of Sport Sciences, 23(6), 561-572.

Rein, R., \& Memmert, D. (2016). Big data and tactical analysis in elite soccer: future challenges and opportunities for sports science. SpringerPlus, 5(1), 1-13.

Ruiz-Ruiz, C., Fradua, L., Fernández-García, Á., \& Zubillaga, A. (2013). Analysis of entries into the penalty area as a performance indicator in soccer. European Journal of Sport Science, 13(3), 241-248.

Sanmiguel-Rodríguez, A. (2020). El mejor FC Barcelona de la historia: un análisis observacional de la influencia de las zonas de finalización de su juego. Revista de CIHEFE, 118, 1-11.

Tamarit, X. (2010). ¿Qué es la periodización táctica? Editorial McSport, Barcelona, España.

Tierney, P. J., Young, A., Clarke, N. D., \& Duncan, M. J. (2016). Match play demands of 11 versus 11 professional football using Global Positioning System tracking: Variations across common playing formations. Human Movement Science, 49, 1-8.

Yin, R. (1984). Case study research: design and methods, applied social research methods series. SAGE Publications, Newbury Park, California

Wright, C., Atkins, S., Polman, R., Jones, B., \& Sargeson, L. (2011). Factors associated with goals and goal scoring opportunities in profesional Soccer. International Journal of Performance Analysis in Sport, 11(3), 438449. 